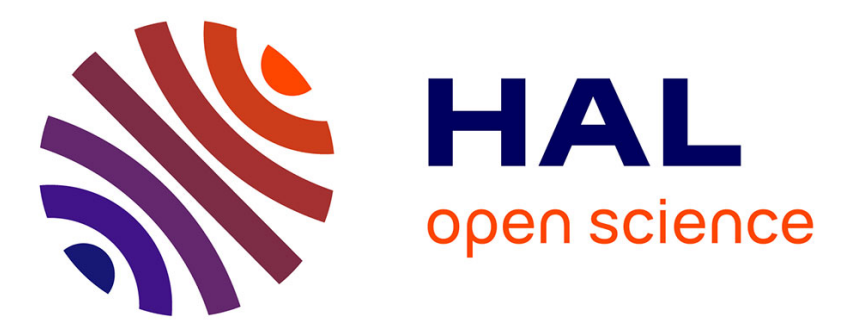

\title{
Pour un relativisme interactionniste conséquent : de Piaget à Darwin et retour
}

\author{
L. Salvador
}

\section{To cite this version:}

L. Salvador. Pour un relativisme interactionniste conséquent: de Piaget à Darwin et retour. Intellectica - La revue de l'Association pour la Recherche sur les sciences de la Cognition (ARCo), 1993, 16 (1), pp.101-131. 10.3406/intel.1993.1429 . hal-03258426

\section{HAL Id: hal-03258426 \\ https://hal.science/hal-03258426}

Submitted on 11 Jun 2021

HAL is a multi-disciplinary open access archive for the deposit and dissemination of scientific research documents, whether they are published or not. The documents may come from teaching and research institutions in France or abroad, or from public or private research centers.
L'archive ouverte pluridisciplinaire HAL, est destinée au dépôt et à la diffusion de documents scientifiques de niveau recherche, publiés ou non, émanant des établissements d'enseignement et de recherche français ou étrangers, des laboratoires publics ou privés.

\section{(이) $\$$}

Distributed under a Creative Commons Attribution - NonCommercial - NoDerivatives| 4.0 


\section{Pour un relativisme interactionniste conséquent : de Piaget à} Darwin et retour

\section{Luc-Laurent Salvador}

\section{Citer ce document / Cite this document :}

Salvador Luc-Laurent. Pour un relativisme interactionniste conséquent : de Piaget à Darwin et retour. In: Intellectica. Revue de l'Association pour la Recherche Cognitive, n¹6, 1993/1. Biologie et cognition. pp. 101-131; doi : https://doi.org/10.3406/intel.1993.1429

https://www.persee.fr/doc/intel_0769-4113_1993_num_16_1_1429

Fichier pdf généré le 29/03/2019 


\begin{abstract}
Taking Interactionist Relativism Seriously, from Piaget to Darwin and back

As the problematic of morphogenesis is a growing point in the field of cognitive sciences, it seems pertinent to focus once again on the genetic conceptions of Piaget, which have been progressively neglected since the booming of the information processing paradigm. This is the main direction of my argumentation which, moreover, will be an attempt to give a better coherence to the hard core of Piaget's theory. I will consider this theory in the light of the original darwinian conceptions. It will become apparent that the empirical difficulties regarding the question of the equilibration of cognitive structures might have been avoided, if Piaget had remained a faithful relativist interactionist. Because of his prejudice towards the autonomy of the elaboration process of the intellectual structures, Piaget has not been able to fully assimilate the darwinian, selectionist and, most of all, non-problematic conception of his "maître à penser", Baldwin.
\end{abstract}

\title{
Résumé
}

Au regard de l'importance croissante de la problématique morphogénétique au sein des sciences cognitives, il serait opportun de réactualiser les conceptions génétiques de Piaget qui ont été progressivement délaissées depuis l'avènement du paradigme computo-symbolique. Tel sera le sens de l'argumentation développée ici. Elle visera, ce faisant, à redonner une meilleure cohérence au noyau dur de la théorie piagétienne. Cette dernière sera examinée en détail, à la lumière d'un darwinisme ressourcé. II apparaîtra que les revers empiriques relatifs à la question de l'équilibration des structures cognitives auraient pu être évités si Piaget s'en était tenu strictement à son relativisme interactionniste. Du fait de ses préjugés quant à l'autonomie du processus de construction des structures intellectuelles, Piaget n'a pas su faire totalement siennes les positions darwiniennes, sélectionnistes et surtout, non-problématiques, de son maître à penser, Baldwin. 
Intellectica, 1993/1, 16, pp. 101-131

\section{Luc-Laurent SALVADOR}

\section{Pour un relativisme interactionniste conséquent — de Piaget à Darwin et retour}

\section{Introduction}

\section{I.1 L'enjeu ontogénétique}

Même si Piaget, disparu depuis une décennie, apparaît toujours comme une référence incontournable, - quelques singuliers historiques de la psychologie cognitive font néanmoins exception (cf. Tiberghien 1986, 1992, Simon 1989) —, il est manifeste que son imposant édifice théorique ne suscite plus le même intérêt que par le passé, alors que l'effervescence va croissant dans le domaine des sciences cognitives. Pour expliquer cet état de fait, on pourrait être tenté d'invoquer la taille, la complexité et peut-être le flou de l'œuvre. Mais, c'est probablement l'alternative offerte par ce qu'il est convenu d'appeler le paradigme STI ou computoreprésentationnel qui en est responsable au premier chef. Et cela, ne serait-ce que par le nouvel espace qui s'ouvrait à ceux qui, las des ukazes piagétiennes, ne voulaient pas s'enfermer dans un système dont les faiblesses se faisaient insistantes. Quelque salutaire qu'ait pu être cette prise de distance par rapport à la théorie piagétienne, on peut toutefois se demander si nous ne verserions pas, en ce moment, dans l'excès contraire :

"Jugés dans la perspective du constructivisme épistémologique et psychologique, une grande partie des travaux contemporains sur des phénomènes tels que la perception, l'image ou la mémoire, manifestent un réalisme désarmant" (Ducret, $1990: 150$ ).

N'aurait-on pas, en quelque sorte, bradé un peu trop vite l'héritage piagétien ? C'est ce que donne à penser l'omniprésence 
actuelle de la problématique de l'évolution, de l'adaptation, dans l'ensemble des sciences cognitives. Effectivement, il est loin le temps où Newell et Simon essayaient de concevoir des architectures cognitives devant fonctionner directement au niveau "expert" ou "adulte". On a depuis pris conscience, tant en intelligence artificielle (Ganascia 1989, Pitrat 1990), qu'en psychologie cognitive computo-symbolique (Cauzinille 1988, Bastien 1989), que l'enjeu premier était la modélisation de capacités d'apprentissage, donc la modélisation de processus d'évolution, d'adaptation. Pour constater le chemin parcouru, il n'est que de citer Simon lui-même :

"Intelligence is closely related with adaptivity - with problem solving, learning, and evolution. A science of intelligent systems has to be a science of adaptive systems with all this entails for the difficulty of finding genuine invariants. Some of the invariance in intelligence is imposed by the structure of the inner environment, for example, the limits of human short-term memory. Some of it is imposed by the outer environment the need to search very large spaces selectively. Some of the invariance is to be found in the structure of learning systems rather than on the highly adapted performance systems they produce. But in cognitive science we must be prepared to recognize that the invariants in an adaptive system are likely to be limited to specific times and places and that in the long run almost any aspect of them can change adaptively" (in Posner, $1989: 43$ ).

Dans le contexte de cet enjeu ontogénétique, toujours plus d'actualité, et où l'intelligence se voit toujours davantage associée à l'adaptation, il me semble que Piaget, inlassable penseur des mécanismes de l'adaptation biologique et cognitive, a proposé des conceptions qui restent d'une extrême pertinence. Notre visée sera ici de prendre ces conceptions au sérieux, c'est-à-dire, à la racine, pour entendre et discuter ce qu'elles ont à nous dire en définitive sur les mécanismes de l'adaptation, sur leur invariants. Pour ce faire, nous allons cibler directement le noyau (pas complètement) dur de l'édifice piagétien, à savoir, les concepts architectoniques d'assimilation/accommodation ${ }^{1}$. Il s'agit là de l'expression typiquement piagétienne du doublet organisation/adaptation, que l'on retrouve sous diverses apparences dans l'ensemble des

${ }^{1}$ Concepts pour lesquels Piaget est redevable tant à Baldwin, qu'à Le Dantec qui déjà fin XIXe désignait l'assimilation fonctionnelle comme la caractéristique essentielle de la vie. 
sciences cognitives (cf. par exemple Grossberg (1987) et sa problématique connexionniste en terme de stabilité/plasticité). Proposés par Piaget dès ses premiers travaux, ces concepts n'ont, à proprement parler, jamais été discutés en tant que tels et, c'est à cela précisément que nous allons nous atteler. En les contestant, en les replaçant dans une perspective darwinienne qui leur a toujours fait défaut, nous tenterons de montrer leur grande actualité relativement aux nouvelles préoccupations "évolutionnaires" et "sub-logiques" des sciences cognitives.

Si nous sommes amené à remonter ainsi jusqu'aux fondations, c'est-à-dire jusqu'au noyau théorique directement lié à l'expérience et la formation de biologiste de son auteur, ce n'est pas par goût pour la scolastique, mais parce que dans une étude précédente sur l'évolution menée sur une base tout à la fois darwinienne et systémique nous avions retrouvé un processus homologue à ce que Piaget appelait l'équilibre entre assimilation et accommodation, équilibre qui était pour lui le critère de l'adaptation. Or, l'essentiel des critiques de la théorie piagétiennc a porté sur ce mécanisme dit de l'équilibration, avec d'importants résultats empiriques qui l'ont sérieusement ébranlée (cf. infra). $\mathrm{Et}$, le fait que Piaget n'ait jamais su y répliquer de manière satisfaisante malgré des tentatives répétées, pourrait, me semble-til, s'interpréter comme la conséquence d'un anti-darwinisme obstiné qui l'aura tenu à l'écart d'une solution systémique, interactionniste mais, par malheur, foncièrement darwinienne.

Outre cet objectif premier qui sera d'inspecter les fondations piagétiennes et, incidemment, d'y rapporter la pierre darwinienne rejetée par le bâtisseur, nous serions pleinement satisfait si, au bout du compte, le lecteur voulait seulement envisager l'éventualité que les sciences cognitives puissent tirer profit d'une ouverture à la perspective piagétienne. Cela, me semble-t-il, essentiellement parce qu'il s'agit d'une conception qui (1) depuis toujours cherche à expliquer, modéliser, simuler, non seulement le fonctionnement instantané de l'organisation, mais aussi et surtout sa genèse ; (2) et qui, insistant - avec maintes hésitations, il est vrai - sur la nécessité de ne pas entretenir de coupure épistémologique entre organisme et environnement, entre le sujet et l'objet, fournit, avec la notion de cycle ou de schème assimilateur un cadre conceptuel parfaitement adapté, tant au 
contexte épistémologique qu'à l'enjeu ontogénétique actuel ; je pense ici à la tentative néo-connexionniste pour générer un niveau symbolique sur une base seulement sub-symbolique, pour produire une "syntaxe d'attracteur", ainsi qu'à cette branche dissidente de l'I.A. "classique" récemment crée et dénommée A(rtificial) LIFE (cf. Langton 1989, 1991).

Après cette perspective d'ensemble, affinons le contexte théorique de notre enquête afin d'en mieux saisir l'objet.

\section{I.2 Le projet piagétien : l'épistémologie génétique}

Piaget, il faut le rappeler, ne s'est consacré à la psychologie de l'enfant que dans le contexte d'une quête épistémologique. Il visait donc à comprendre comment s'élabore la connaissance et, ayant adopté la position commune aux lamarckiens - comme à certains darwiniens tel Baldwin - quant au rôle déterminant de l'ontogenèse dans l'évolution, il pensait capturer les invariants du processus de construction des connaissances en étudiant comment, chez l'enfant, l'élaboration de ses structures intellectuelles lui permet de rejoindre les grandes catégories de la pensée.

Mais ses recherches de psychologie génétique, entamées au tout début des années 20 , pour avoir eu l'audience que l'on sait, n'en ont pas moins achoppé sur d'insurmontables problèmes. La difficulté essentielle résidant dans son modèle de l'équilibration. Ce modèle devait rendre compte du processus par lequel l'organisation, tant biologique qu'intellectuelle (les deux étant cognitives de bout en bout) pouvait se construire au travers de son fonctionnement au sein d'un environnement avec lequel elle entretenait une constante adaptation, c'est-à-dire un équilibre dans les échanges organisation/milieu, ces échanges se faisant toujours plus riches et plus complexes au cours du développement. Il s'agissait, en bref, de concevoir un modèle de construction d'une organisation au travers des interactions qu'elle mène avec son environnement. De fait, Piaget se disait constructiviste, relativiste interactionniste. Sur la base de telles options, il cherchait à échapper aux inévitables oppositions telles que par exemple idéalisme/réalisme, innéisme/empirisme, darwinisme/lamarckisme et qui sont toutes relatives au primat donné aux facteurs internes 
ou aux facteurs externes, dans l'explication de l'organisation (biologique ou cognitive).

Piaget cherchait à établir un tertium, c'est-à-dire à transcender cette classique coupure entre le sujet et l'objet et pour cela pensait pouvoir faire appel tout à la fois à l'activité du sujet dans son environnement et à son activité interne de mise en cohérence de sa structure.

I.3 Les difficultés de l'autonomie : le recours aux "perturbations"

Cette position toutefois ne pouvait manquer de déboucher sur les difficultés inhérentes à la juxtaposition de deux modes causaux. Qu'est ce qui en dernier ressort est responsable de l'élaboration des structures intellectuelles ? La dynamique des interactions avec l'environnement ou le travail interne de mise en cohérence ? A question mal posée, réponse impossible ! Piaget n'a effectivement pas su tenir son relativisme interactionniste jusqu'au bout. Il était avant tout résolu à ne pas laisser de responsabilités à l'environnement dans l'élaboration des structures intellectuelles qui, par conséquent, n'évolueraient que sous l'effet de la mise en cohérence (l'abstraction réfléchissante et/ou assimilation réciproque) qu'elles-mêmes déterminent. Dès lors, il s'est vu acculé au paradoxe que les penseurs de l'autonomie cybernétique ont eu à gérer à la suite du fameux théorème d'Ashby (1962) établissant qu'il ne saurait y avoir d'autonomie véritable.

Et, il est très remarquable de constater que la pseudo-solution de Piaget est tout à fait analogue à celles développées par Atlan (1972, 1979) ou Varela (1989), en ce qu'elle fait aussi appel aux perturbations. Si vous n'admettez pas qu'une information externe vienne directement in-former une organisation et si vous admettez avec Ashby que cette organisation ne saurait davantage se structurer elle-même que vous ne pouvez vous envoler en tirant sur vos laçets, il ne vous reste qu'à admettre que "quelque chose" d'extérieur, qui serait sans signification, vient perturber ladite organisation, la déséquilibrer, entraînant de ce fait un processus de rééquilibration déterminé, quant à lui, par les seules caractéristiques de l'organisation et débouchant sur un nouvel équilibre. 
Ce que nous allons tenter de montrer en définitive, c'est que cette fragile conception "autonomiste" dans laquelle Piaget s'est enfermé est inutile pour autant qu'on veuille bien s'abstenir fermement de rétablir, à quelque moment que ce soit, une coupure épistémologique entre le sujet et l'objet, l'organisation et l'environnement. Ce qu'à l'évidence, Piaget n'a pas su faire, soucieux qu'il était de défendre l'autonomie du sujet.

\section{I.4 Plan}

Pour ce faire, nous effectuerons, tout d'abord, une rapide présentation du concept de schème, schème assimilateur qui, dans sa seule définition, recueille l'essence même du système piagétien. Puis nous nous focaliserons sur la pierre d'achoppement de ce système : l'accommodation, présentée comme l'effet des pressions exercées par le milieu, ce qui est, on l'a vu, extrêmement paradoxal de la part d'un auteur se défiant de tout empirisme. Effectuant alors un retour à la théorie darwinienne originelle, nous exposerons rapidement quelques éléments de la conception dite "écosystémique" de l'évolution développée dans une précédente étude. Celle-ci, immédiatement transposable en écosystémique des schèmes, nous fera retrouver les schémas piagétiens, mais dans une version darwinienne, cohérente, qui permet de penser l'équilibration hors de toute aporie.

Notre conclusion consistera, en quelque sorte, à tirer la morale de l'échec piagétien pour défendre une conception processuelle de l'ontologie dont, paradoxalement, les concepts piagétiens me semblent le meilleur fondement.

\section{Le schème piagétien}

\section{II.1 Un cycle fonctionnel}

Bien que, du fait de son ubiquité, on puisse être tenté de présenter le schème comme la brique du système piagétien, ce serait certainement une mauvaise métaphore car elle ne véhicule pas l'idée essentielle qui se cache derrière cette notion : celle de totalité. Loin d'avoir une pensée qui atomise son champ d'investigation, Piaget s'est attaché, avant toute chose, à respecter 
l'intégrité de son objet d'étude : l'organisation, tant biologique que cognitive. Or, on ne saurait appréhender une organisation en se focalisant sur ses seuls éléments pris séparément, les uns après les autres. C'est tout ce qui fait la différence entre le biochimiste et le biologiste. Pour le premier, il n'y a pas de distinction à faire entre un organisme vivant ou mort.

C'est donc l'essence même de l'organisation que Piaget a voulu d'emblée recueillir dans sa notion de schème. Il a, pour ce faire, donné l'image du cycle de processus, c'est-à-dire d'un enchaînement de processus bouclant sur lui-même, de sorte qu'il se perpétue de par le simple fait qu'il s'exécute. Outre le fait qu'elle n'est pas très novatrice puisqu'elle a déjà été élaborée dans le cadre de la cadre de la biologie théorique de von Uexküll (1926, 1965), cette définition risque surtout d'apparaître par trop minimaliste. Pourtant, il faut constater qu'elle a par la suite, sous diverses formes, tenu un rôle de premier plan, tant dans les recherches biochimiques sur l'origine du code génétique (Eigen $\&$ al. 1981), qu'en chimie, dans la conceptualisation des structures dissipatives (Prigogine \& al. 1971). Prigogine et Piaget étaient d'ailleurs bien au fait de cette similitude (cf. Inhelder \& al. 1977). De même, dans la définition qu'il donne de l'organisation du vivant comme processus autopoiétique, Varela fait, lui aussi appel à cette conception :

"Un système autopoḯtique est organisé comme un réseau de processus de production de composants qui (a) régénèrent continuellement par leurs transformations et leurs interactions le réseau qui les a produits, et qui, (b) constituent le système en tant qu'unité concrète dans l'espace où il existe, en spécifiant le domaine topologique où il se réalise comme réseau." (Varela, $1989: 45$ ).

Le deuxième point de cette citation nous amène maintenant à la question cruciale des rapports que peuvent entretenir de tels "cycles" avec leur environnement. Pour Piaget, il est d'emblée évident que cette chaîne de processus, pour fermée qu'elle soit, n'en est pas moins indissociable du milieu dans lequel elle s'exécute. Et par conséquent, il nous propose une représentation parfaitement symétrique, où cycle et milieu sont aussi peu dissociables l'un de l'autre que peut l'être un tourbillon de l'eau dans laquelle il a pris naissance : 
"Appelons A,B,C...Z les éléments, matériels ou dynamiques, d'une structure comportant un ordre cyclique, et $\mathrm{A}^{\prime}, \mathrm{B}^{\prime}, \mathrm{C}^{\prime}, \ldots \mathrm{Z}^{\prime}$ les éléments, matériels ou énergétiques, nécessaires à leur entretien. On aura alors, si le signe * représente l'interaction des termes du premier ensemble et de ceux du second, et si le signe $\rightarrow$ représente l'aboutissement de ces interactions :

$$
\left(A^{*} A^{\prime}\right) \rightarrow\left(B * B^{\prime}\right)->\left(C^{*} C^{\prime}\right) \rightarrow \ldots\left(Z^{*} Z^{\prime}\right) \rightarrow\left(A * A^{\prime}\right)->\text { etc. }
$$

En un tel cas, on est en présence d'un cycle fermé en tant que cycle et exprimant la reconstitution permanente des éléments $A, B, C . . Z, A$, etc. qui caractérisent les parties de l'organisme.." (Piaget, 1967 : 183).

Certes, cette notation ne saurait empêcher qui que ce soit de persévérer dans une volonté de distinction, car on trouve bien des éléments qui sont présentés comme appartenant en propre au cycle et d'autres qui se trouvent être ceux du milieu. Ce serait, ce faisant, en méconnaître complètement l'esprit. J'en veux pour preuve ce qu'écrivait Le Dantec, véritable inventeur ${ }^{2}$ de ladite notation :

"Un être vivant n'est entièrement défini comme mécanisme que si l'on fait entrer en ligne de compte, au moment où on le considère, ses relations actuelles avec les agents physico-chimiques de son ambiance. J'ai proposé jadis de rappeler cette particularité par un symbolisme simple : si l'on représente par A la structure totale d'un être vivant à un moment précis de son existence, et par B, l'ensemble des facteurs du milieu ambiant avec lesquels il est relation à ce moment précis, ce qui caractérise le fontionnement de l'organisme au moment considéré, ce n'est ni A tout seul, ni B tout seul, mais bien A et B a la fois, et surtout, les relations actuelles entre $A$ et $B$, relations que je represente par le symbole $(A * B)$. Ce symbole $\left(\mathrm{A}^{*} \mathrm{~B}\right)$ représente tous les éléments qui interviennent dans l'activité de l'individu au moment considéré, et définit, par conséquent, son fonctionnement actuel tout entier.

M. Et. Rabaud a adopté le même symbolisme dans un livre récent et a substitué à la narration ordinaire des physiologistes qui font de l'animal le sujet du verbe actif dans la phrase, un langage dans lequel on ne parle ni de l'animal seul ni du milieu seul, mais du complexe "organisme*milieu". Je crois qu'il est impossible de se passer de cette formule symbolique, si l'on veut parler un langage clair et vraiment scientifique." (Le Dantec, $1913: 81)$.

2 Bien qu'il ne soit pas de notre propos de faire ici l'histoire de la pensée piagétienne, il est clair que le moment venu, c'est à une relecture serrée de Le Dantec qu'il faudra s'atteler. Les homologies telles que celle que nous venons de relever sont légion. Il semble bien que César lui-même eût dû, parfois, rendre à César... 


\section{II.2 Assimilation et accommodation}

Ce cycle, Piaget l'appelait le cycle assimilateur pour la bonne raison qu'il instancie, essentiellement, un processus qui se perpétue en "intégrant" constamment des éléments du milieu que l'on peut considérer comme les "aliments" du cycle. Ces "aliments", en étant intégrés, assimilés, autorisent le déroulement, le fonctionnement du cycle et donc, sa fermeture et sa stabilité. On voit ainsi que l'assimilation est corrélative d'une organisation préalable à l'interaction. Rien n'est assimilé qui ne le soit par une organisation et aucune organisation ne se perpétue autrement qu'en assimilant les éléments "externes" nécessaires à son entretien. Observons maintenant qu'à chaque étape de son exécution, cette organisation minimale instancie une "anticipation" quant à sa prochaine interaction avec l'environnement, une "anticipation" de l'élément du milieu qui lui permettra de fonctionner, d'assurer sa fermeture, sa stabilité. Considérons alors le cas où un $A^{\prime \prime}$ se présente au lieu du $A^{\prime}$ habituel, deux cas de figure en découlent :

a) ou bien le cycle ne peut se fermer, il ne s'adapte pas et se désorganise.

b) ou bien le cycle s'adapte et par exemple :

$\left(\mathrm{A}^{*} \mathrm{~A}^{\prime \prime}\right)$ donne $\mathrm{B} 1$ au lieu de $\mathrm{B}$, puis $\left(\mathrm{B} 1^{*} \mathrm{~B}^{\prime}\right)$ donne $\mathrm{C}$; le cycle, accommodé, se poursuivant ensuite normalement. Mais on pourrait aussi imaginer que le cycle ne récupère son équilibre qu'après une cascade de perturbations rattrapées.

Piaget s'en est toujours tenu à ces deux possibilités, mais il conviendrait d'ajouter le cas où $\left(A^{*} A^{\prime \prime}\right)$ redonne tout bonnement $B$. Cela signifierait que l'organisation du cycle n'est pas en mesure de distinguer $A^{\prime}$ de $A^{\prime \prime}$. $A^{\prime}$ et $A^{\prime \prime}$ seraient assimilés au même schème ; ils seraient ainsi assimilés l'un à l'autre, non distingués.

Dans le cas où le cycle s'adapte, on parle d'accommodation, cette dernière apparaissant ainsi comme la conséquence de la résistance du milieu à son assimilation.

$$
\text { II.3 Cycle }=\text { schème }=\text { holon }=\text { intégron }
$$

Ce tableau du cycle assimilateur a été brossé en termes non spécifiques pour la bonne raison qu'il gardera sa pertinence à 
quelque niveau que ce soit. Ainsi, son domaine va du niveau purement biologique - avec par exemple l'assimilation physiologique dont Le Dantec s'est précisément inspiré pour concevoir la notion générale d'assimilation fonctionnelle - au niveau intellectuel, en passant par les organisations comportementales plus ou moins complexes comme la succion, la préhension, la marche etc. En pratique, dès que l'on n'a plus affaire à des énoncés de principes illustrés au niveau physicochimique, c'est-à-dire dès le niveau comportemental, Piaget parle de schème plutôt que de cycle.

Cette ubiquité est autorisée par le caractère récursif de la définition, qui n'est pas aussi simpliste qu'il y paraît : effectivement, chacun des éléments du cycle peut être lui aussi un cycle. Le schème est ainsi l'équivalent du holon de Koestler (1979), ou de l'intégron de Jacob (1970), tout à la fois une totalité composée d'éléments qui peuvent aussi être des schèmes et l'élément d'une totalité englobante qui est aussi un schème, de niveau supérieur.

"Il y a organisation à l'intérieur de chaque schème d'assimilation, puisque chacun constitue un tout réel, conférant à chaque élément une signification relative à cette totalité. Mais il y a surtout organisation totale, c'est-à-dire coordination entre les schèmes divers d'assimilation." (Piaget, $1967: 130$ ).

II.4 L'interaction organisation/environnement : vers l'équilibre?

Concentrons-nous à présent sur la partie la plus difficile et aussi la plus lourde de signification : l'entre-deux, l'interface organisation/environnement. Ici, ainsi qu'on vient de le voir, ces interactions sont présentées sous deux aspects :

- Un aspect conservatif, de stabilisation, au sens où les interactions autorisent le fonctionnement et la fermeture de l'organisation, donc sa perpétuation : c'est l'assimilation. L'organisation assimile les éléments - les aliments - nécessaires tout à la fois à son fonctionnement et à son maintien puisque, nullement statique, elle est constamment mise en jeu et reconduite (ou non) à chaque étape de son exécution, à chaque étape de l'assimilation. 
- Un aspect évolutif ou adaptatif, relatif aux modifications "subies" par le schème du fait des pressions $d u$ milieu, de ses résistances à l'assimilation :

"la constitution des schèmes par l'assimilation implique l'utilisation de réalités extérieures auxquelles force est à ceux-là de s'accommoder si grossièrement que ce soit." (Piaget, 1937 : 309).

Ces deux volets revêtent immédiatement une dimension cognitive, en ce sens que :

$\left.1^{\circ}\right)$ Le cycle, le schème, l'organisation ne retient, n'intègre du milieu que les éléments qui participent de son fonctionnement. De ce fait, on peut, avec Piaget, considérer que l'organisation s'impose au milieu, au sens où, en n'en retenant que ce qui entre dans ses cadres, elle génère, spécifie, un "environnement" qui lui est propre. Uexküll ne cherchait pas à exprimer autre chose avec sa notion d'Umwelt.

$2^{\circ}$ ) Ce sont les résistances qu'offre le "réel" à son assimilation qui entraînent l'accommodation du schème, de l'organisation.

Paradoxal, car présenté dans le contexte d'un relativisme interactionniste, cet appel aux résistances du réel voit cependant ses relents d'empirisme vite masqués. En effet, Piaget affirme d'emblée que l'adaptation - c'est-à-dire au niveau intellectuel, l'intelligence - ne saurait être définie par la seule accommodation :

"Il convient d'abord d'expliquer pourquoi nous ne définissons pas l'adaptation par l'accommodation seule, comme on pourrait être tenté de le faire. La raison en est que sans assimilation, il n'y a pas adaptation au sens biologique du terme : on peut, par exemple, dire métaphoriquement qu'un liquide adapte sa forme à celle du récipient, mais il n'y a là aucune adaptation biologique, car cette forme nouvelle ne constitue qu'un accident momentané et ne se conservera pas en cas de nouveau transvasement, faute précisément de toute assimilation à une organisation permanente." (Piaget, 1967 : 202).

De la sorte, Piaget signifie clairement que l'impression de la "cire vierge", pas davantage que l'accommodation de l'eau à son récipient, ne constituent des "instances" d'un processus d'adaptation car l'assimilation fait défaut : 
"une accommodation ne peut être que l'accommodation d'une structure organisée et ne peut donc se produire, sous l'influence d'un facteur ou élément extérieurs, que dans la mesure où il y a par ailleurs assimilation momentanée ou durable de cet élément ou de son prolongement à la structure qu'il modifie." (Piaget, 1967 : 202).

D'où cette idée, invariablement soutenue, qu'il convient de définir l'adaptation par l'équilibre entre assimilation et accommodation - et non par cette dernière seulement :

"l'adaptation intellectuelle, comme tout autre, est une mise en équilibre progressive entre un mécanisme assimilateur et une accommodation complémentaire." (Piaget, $1936: 13$ ).

\section{II.5 La construction nécessaire des connaissances}

Tout cela parait bel et bon et n'a, à ma connaissance, jamais fait l'objet de discussions ou de critiques. Pas à ce niveau du moins, car, par ailleurs, on n'a cessé de réfléchir sur la notion d'équilibration qui a tenu une place cruciale dans l'explication du processus d'élaboration des structures intellectuelles, particulièrement au niveau des opérations logiques. Piaget a toujours voulu considérer ce processus comme autonome ou autoréglé. Il refusait l'idée que l'abstraction empirique - ce que nous pouvons tirer des objets en les assimilant - puisse intervenir autrement que comme aliment des processus de l'abstraction réfléchissante - le travail de mise en cohérence par assimilation réciproque entre schèmes. Il concevait donc la filiation des structures intellectuelles sur le mode de l'enchaînement logique et nécessaire d'une construction mathématique, ce qui a été établi précédemment guidant la construction de ce qui suit ; cet enchaînement étant, à chaque étape, vu comme la conséquence d'un processus d'équilibration. D'où sa totale approbation de Waddington lorsque ce dernier illustrait le processus de l'épigenèse par l'image d'un enchaînement de théorèmes (cf. Piaget, $1967: 24)$.

Il importe de rappeler ici que même s'il ne faut pas, évidemment, rapprocher sans précaution l'équilibration "physicochimique" de celle relative au domaine logico-mathématique, l'assimilation et l'accommodation constituent bien, selon Piaget, des invariants fonctionnels communs aux domaines biologique et 
cognitif. Ainsi, dans quelque domaine que l'on considère l'équilibration, ce sont toujours les mêmes fonctions générales qui sont à l'œuvre, elles sont seulement dans un contexte différent. Dès lors, il y a certainement quelques indications de portée générale à tirer des revers empiriques que Piaget a connu à ce niveau logico-mathématique et nous allons rapidement en traiter.

\section{II.6 Le primat assimilateur et les décalages horizontaux}

Le caractère nécessaire, donc endogène et autonome, de cette filiation des structures intellectuelles est d'abord apparu constestable par la mise en évidence d'un effet des situations d'apprentissage auxquelles les sujets étaient soumis. Mais, c'est surtout l'analyse du phénomène dit des décalages horizontaux qui $a$, me semble-t-il, amené les résultats les plus intéressants en interdisant l'appel aux résistances des objets, aux perturbations qu'ils sont censés susciter.

Rappelons encore que selon Piaget, toute organisation se construit au travers de l'activité qu'elle déploie au cours de son fonctionnement. Par conséquent, c'est à l'organisation qu'il convient d'accorder un primat, en ne laissant à l'environnement qu'un minimum de responsabilités. L'environnement n'étant de toute manière défini que relativement aux capacités d'assimilation de l'organisation, l'abstraction empirique serait de fait déterminée par l'organisation préalable à l'interaction, donc par les effets de l'abstraction réfléchissante opérée par le sujet.

Les problèmes ont découlé de ce primat assimilateur, car, au niveau logique, il devait se traduire particulièrement par le fait qu'une fois une structure intellectuelle élaborée, elle devait pouvoir s'appliquer à n'importe quel domaine du réel. Les expérimentations ont montré que ce n'était pas le cas. Cela s'est traduit par des décalages horizontaux, c'est-à-dire par le fait que les sujets, dans certaines situations, peuvent, par leur comportement et leurs commentaires, manifester certaines structures intellectuelles qu'ils ne savent mettre en oeuvre en d'autres situations. C'est ici que Piaget en appelaient de manière quelque peu ad hoc aux inévitables singularités, aspérités des objets sur lesquels le sujet opère et qui génèreraient un "frottement" responsable des décalages d'une situation 
expérimentale à l'autre, d'un domaine à l'autre. Cependant, de récentes études de psychologie différentielle (qui analysent la variabilité inter et intra-individuelle plutôt que de la moyenner) ont montré que le sens des décalages variait selon les sujets et que, par conséquent, on peut difficilement invoquer les singularités de l'objet car les décalages devraient, alors, tous être de même sens (Lautrey (1987, 1990), Bideaud (1989)). Cette impossibilité d'en référer aux résistances de l'objet, à ces perturbations indispensables aux conceptions "autonomistes", replonge la théorie de Piaget dans le paradoxe de l'équilibration. Ces résultats, en obligeant la théorie à s'en tenir à ses seuls principes "autonomistes", la remettent en demeure d'expliquer comment les structures cognitives pourraient s'enrichir par le seul travail de mise en cohérence interne, le domaine empirique n'étant, en principe qu'un simple support pour l'activité ou les opérations desdites structures. Le bruit organisateur n'étant plus de mise, le boostrapping reste un mystère.

Lautrey, s'accordant en cela avec une conception esquissée indépendamment par Henriques (Inhelder \& al., 1977 : 123), pense pouvoir faire fonds sur le polymorphisme des processus mentaux, considérant que le "propositionnel" pourrait se voir "ensemencé", "guidé" par le "figuratif" et réciproquement. Mais cette solution n'est pas complètement satisfaisante dans la mesure où elle ne fait que reculer le problème : s'envoler à deux, en se tirant ou en se poussant mutuellement n'a pas de raisons d'être longtemps plus effectif que lorsqu'on est seul à tirer sur ses lacets ou ses tirants de bottes (bootstrapping). Tôt ou tard, il manque des points d'appui.

C'est, par conséquent, la position même du problème qui est à revoir. Il faut se pencher plus avant sur le noyau théorique, là précisément où il est fait appel aux perturbations, aux résistances du réel, dans la définition même de l'accommodation.

\section{Le problème de l'accommodation}

Quand on relit Piaget dans cette perspective, à la recherche de l'argumentation relative au caractère indissociable de l'assimilation et de l'accommodation, on ne trouve rien d'autre que le rappel de cette évidence qui veut que l'accommodation soit 
toujours l'accommodation de quelque chose, donc d'une organisation assimilatrice. Si nous pouvons admettre sans réserve que l'accommodation présuppose l'assimilation, par contre, il ne va pas de soi que l'accommodation doive accompagner l'assimilation et, sur ce point, l'argumentation se fait pernicieuse :

"L'assimilation ne peut jamais être pure, parce qu'en incorporant les éléments nouveaux dans les schèmes antérieurs, l'intelligence modifie sans cesse ces derniers pour les ajuster aux nouvelles donnees." (Piaget, $1936: 13)$.

Cette assertion, censée expliquer pourquoi l'assimilation doit toujours déboucher sur l'accommodation, présuppose insidieusement l'accommodation puisqu'il s'agit d'emblée d'incorporer des éléments nouveaux. Ce qui revient à dire que l'assimilation n'est jamais pure puisqu'il faut accommoder. C'est tout de même un peu facile. On le verra plus loin, Piaget est malheureusement coutumier du fait - quand il ne fait pas purement acte d'autorité.

Quant à l'équilibre entre assimilation et accommodation, il est généralement posé à la suite des considérations précédentes et semble en découler naturellement. Rien ne venant en justifier la nécessité, il ne relève en fait que du dogme, ou au mieux de la définition.

Cet état de fait sera finalement reconnu en tant que tel, puisque dans sa dernière mouture, l'ensemble va se retrouver présenté explicitement sous forme de postulats :

"Premier postulat : Tout schème d'assimilation tend à s'alimenter, c'està-dire à s'incorporer les éléments extérieurs à lui et compatibles avec sa nature. Ce postulat se borne à assigner un moteur à la recherche, donc à considérer comme nécessaire une activité du sujet, mais il n'implique pas par lui-même la construction de nouveautés, car un schème assez large (comme celui "d'êtres") pourrait s'assimiler tout l'univers sans modifier celui-ci ni s'enrichir lui-même en compréhension.

Deuxième postulat : Tout schème d'assimilation est obligé de s'accommoder aux éléments qu'il assimile, c'est-à-dire de se modifier en fonction de leurs particularités, mais sans perdre pour autant sa continuité (donc sa fermeture en tant que cycle de processus interdépendants), ni ses pouvoirs antérieurs d'assimilation. Ce second postulat (déjà valable au plan biologique avec la formation des "accommodats" phénotypiques) affirme la nécessité d'un équilibre entre l'assimilation et l'accommodatiion 
pour autant que l'accommodation réussit et reste compatible avec le cycle, modifié ou non... Notons surtout que, si le second postulat conduit à exiger la formation d'un équilibre entre l'assimilation et l'accommodation, cela n'implique rien de plus que d'affirmer : 1) la présence nécessaire d'accommodations dans les structures de cycles, et 2) la conservation de telles structures en cas d'accommodations réussies." (Piaget, 1975 : 13) (c'est moi qui souligne).

Pour être la base de son ultime version de la théorie de l'équilibration, cet ensemble de définitions n'en contient pas moins une somme surprenante d'incongruités qui, singulièrement, viennent parfaitement illustrer la conception typiquement piagétienne du développement selon laquelle les structures anciennes sont conservées par intégration dans les nouvelles. Effectivement, tout est là, rassemblé, dans sa fragilité originelle, et un livre ne suffirait pas à en commenter tous les aspects car, au fond, c'est bien l'ensemble du système piagétien qui s'y trouve recueilli. Nous irons ici droit à ce qui me semble être l'aspect le plus controversable, à savoir, cette singulière obligation de s'accommoder dans laquelle se trouverait le schème assimilateur.

Cette obligation, radicale puisque posée comme principe, suscite diverses difficultés :

1) La possibilité d'une assimilation pure est de la sorte totalement exclue. Or, en partant du principe même du cycle assimilateur, on est bien en droit de s'attendre à ce qu'un élément qui ne serait pas nouveau - et qui donc, aurait déjà été assimilé - n'entraîne aucune accommodation du cycle, ce dernier l'intégrant sans autre forme de procès. Cette possibilité, Piaget l'admet sans sourciller deux plus tard (Inhelder \& al., 1977 : 59), cn ccla très cohérent avec lui-même, puisqu'il l'avait déjà évoquée dès 1936. Mais, pourrait-on s'exclamer, à quoi tout cela rime-til ? A l'évidence, Piaget est ici instable, hésitant, et semble surtout vouloir soutenir l'idée d'équilibre en dépit des contradictions que cela entraîne avec les définitions du "schématisme" qu'il élabore conjointement. Sans doute espère-t-il les concilier tôt ou tard, et, se refusant à s'amputer de ses intuitions, il s'autorise la contradiction. Il peut ainsi écrire :

"L'esprit ne peut se trouver adapté à une réalité que s'il y a parfaite accommodation, c'est-à-dire si plus rien, dans cette réalité, ne vient modifier les schèmes du sujet". 
et six lignes plus loin,

"Mais toujours et partout, l'adaptation n'est achevée que lorsqu'elle aboutit à un système stable, c'est-à-dire lorsqu'il y a équilibre entre assimilation et accommodation" (Piaget, $1936: 13$ ).

Quels qu'en soient ses motifs, la contradiction est bien réelle. Et elle est d'importance, car, ce qui se joue ici, c'est l'ontologie du cycle, du schème, et donc l'ontologie de tout le système piagétien. La question est, en effet, de savoir si, lorsqu'on se réfère à une entité cycle - ou schème - entendue comme étant dans un état stable, cette stabilité signifie la disparition des transactions régulatrices entre le cycle et son environnement - plus rien ne vient modifier les schèmes du sujet - ou, s'il faut, au contraire, délaisser cette ontologie "objective" pour une ontologie "processuelle" où la stabilité découlerait d'un équilibre dans les interactions du cycle et de son environnement. Nous reviendrons dès que possible sur ce point, pour montrer que, dans un cadre darwinien ou baldwinien, la réponse est évidente, puisque c'est la contradiction elle-même qui est levée.

2) Quelles que soient les velléités affichées par Piaget quant à l'établissement d'un tertium entre idéalisme et empirisme, entre darwinisme et lamarckisme, l'appel aux pressions du milieu, qu'il a copieusement critiqué dans cette dernière doctrine, reste ici la proie des mêmes critiques. Que cela plaise ou non, les pressions du milieu expliquent trop, Piaget lui-même le dit très fermement. Alors pourquoi y revient-il ? Refuser, d'une part, tout ce qui s'apparente à la sélection naturelle et, d'autre part, invoquer les pressions du milieu, n'est certainement pas le plus sûr moyen de construire un tertium!

3) La pire difficulté reste cependant la flagrante contradiction qui découle de la juxtaposition des deux postulats. Le premier dit qu'un schème pourrait s'assimiler tout l'univers sans se modifier, quand le second affirme sans détour que le schème est obligé de s'accommoder. Cette contradiction était en fait présente dès l'origine - et l'on doit savoir gré à Piaget de ne pas avoir, depuis, tenté de l'estomper. Effectivement, sans doute à la suite de ses premières observations de nourrissons, il est amené à déclarer : 
"Le propre d'un schème d'assimilation est de tendre à s'appliquer à tout et conquérir l'univers..dans sa totalité" (Piaget, $1936: 362$ )

ou, plus explicite encore :

"rien ne contraint l'enfant à tenir compte de la multiplicité du réel [...] tant qu'il utilise les objets comme simples aliments fonctionnels" (ibid.).

Il faut voir en cette tendance hégémonique un effet dé la símple exécution du cycle qui amène ce demier à appréhender, à íntégrer tout élément du milieu susceptible de participer de son fonctionnement, tout élément apte à en assurer la fermeture, donc la perpétuation. Le schème se généralise ainsi "spontanément". Une excellente illustration de ce fait est donnée par le bébé qui, à un moment donné, tente d'assimiler tous les objets de son environnement en les suçant. Piaget, d'ailleurs, n'hésite pas à parler de comportement autiste pour bien marquer combien la réalité "objective" est absentée.

Ceci étant, on comprend mal alors comment de tels schèmes pourraient être obligés de s'accommoder ? D'où vient "la nécessité où se trouve l'assimilation de tenir compte des particularités propres aux éléments à assimiler" (Piaget, 1975 : 12) ? Là est la question.

En somme, on ne peut éviter les questions suivantes : comment un schème peut-il s'accommoder alors qu'il s'accommode fort bien de ne pas s'accommoder ? Ou, aussi bien, qu'est-ce qui empêche un schème de délirer, de s'emballer et de tout assimiler ? Et finalement, comment peut-il s'accommoder aux singularités d'un objet lors même que, par principc, il est incapable de les "percevoir" ?

A ces questions, nous allons proposer une réponse, pour découvrir ensuite qu'elle se trouve déjà dans le système piagétien, mais, pour la repérer en tant que telle, c'est-à-dire en tant que solution, il nous aura fallu préalablement effectuer un retour $\grave{a}$ Darwin! 


\section{Evolution écosystémique des espèces}

La place va manquer toutefois pour exposer en détail ce retour à Darwin et, par conséquent, nous nous contenterons d'un rapide survol des quelques conclusions que j'ai pu tirer lors d'une précédente étude (Salvador 1992) à laquelle je renvoie le lecteur intéressé par une argumentation plus étoffée.

En premier lieu, il convient de rappeler le caractère fondamentalement systémique de la conception qu'avait Darwin des processus de l'évolution. Il admettait l'existence d'une causalité circulaire dans les rapports intimes qu'une espèce entretient avec son milieu, et ce, contrairement aux néodarwiniens qui ne voient jamais dans l'évolution que le résultat de la sélection naturelle entendue comme l'action strictement unidirectionnelle de l'environnement sur les êtres vivants (sur le caractère systémique de la pensée darwinienne cf. Gruber 1966, Patten 1980, Lenay 1989). De ce fait, ceux qui critiquent le néodarwinisme, et son fameux doublet "mutation + sélection", devraient y regarder à deux fois avant d'excommunier aussi Darwin. C'est ce que n'a pas su faire Piaget, bien qu'un de ses maîtres à penser, Baldwin, ait par ailleurs été un fervent darwinien - et non pas néo-darwinien.

Pourtant, ce cadre darwinien originel rendait possible une conception du processus évolutif qui, loin de réduire la stabilité et/ou l'évolution des formes vivantes au seul niveau génétique - prenant en compte le couplage, la co-détermination des formes vivantes - aurait montré en quoi la stabilité d'une forme spécifique (mais aussi son éventuelle transformation) résulte d'une dualité de processus en équilibre. Cet équilibre se jouant dans lcs interactions que chaque forme vivante entretient avec le milieu dans lequel elle s'active, milieu qui est, on va le voir, essentiellement constitué par les autres espèces participant de la biocénose.

On peut ainsi montrer que :

1) La stabilité morphologique d'une espèce, l'invariance de la norme spécifique, n'est pas la conséquence, ainsi que le pensait Monod (1970), de la reproduction ne varietur autorisée par les capacités de réplication de l'ADN. C'est maintenant un fait bien établi, il y a une importante variabilité génétique entre les 
individus d'une même espèce et la reproduction sexuée est, justement, un mécanisme qui assure un brassage constant du "pool génétique", de sorte que la reproduction s'effectue toujours sur la base d'un bruit de fond. La norme spécifique a donc inévitablement tendance à se relâcher, à s'élargir ; la stabilité doit donc être expliquée autrement.

2) Une explication s'impose tout naturellement si l'on invoque la possibilité d'une reproduction différentielle consécutive au fait que certains des "variants" les plus éloignés de la tendance centrale de la population sont moins à même de se reproduire correctement, leurs conformations les amenant à interagir avec leur milieu biotique et abiotique avec une efficacité moindre au plan de la reproduction. La différence, même très faible, pourra suffire à "élaguer" la courbe de Gauss de la population et à la maintenir constante plutôt que de la laisser s'étaler.

3) Par conséquent, la cohérence de la biocénose, — c'est-à-dire l'ensemble des formes vivantes participant de cet écosystème constitue le facteur qui, fondamentalement, détermine la stabilité ou l'évolution de l'espèce.

Effectivement, on comprendra aisément que dans une biocénose très dense, très compartimentée comme celle de la forêt tropicale, où les niches écologiques se jouxtent - c'est-à-dire, en somme, lorsque la cohérence est élevée -, il soit difficile pour une espèce donnée d'exprimer sa tendance à s'assimiler l'univers aussi facilement que dans le cas inverse d'une biocénose pratiquement vide, comme cela a été le cas, par exemple, pour les premiers tétrapodes partis à la conquête des terres émergées.

Ce point est d'une importance extrême. Il n'est cependant que l'expression directe de la simple co-détermination, ou causalité circulaire, assumée d'entrée de jeu. Et c'est à cela qu'il va falloir se tenir pour apprécier toute la différence entre les positions de Darwin-Baldwin et Piaget.

\section{V. Évolution écosystémique des schèmes}

Le lecteur l'aura anticipé, en (1) est décrit un processus homologue ou plutôt une excellent exemple de schème assimilateur. On y retrouve bien, d'une part, l'aspect conservateur, puisque l'organisation que constitue - la 
population de - l'espèce se perpétue grâce à la reproduction des individus, et d'autre part, on retrouve la tendance à l'élargissement de l'organisation. Cette dernière se ferait toujours plus ample si le processus décrit en (2), la reproduction différentielle, ne venait l'élaguer. Pour ceux qui ne l'aurait pas reconnue, précisons qu'il s'agit là de ce que l'on appelle traditionnellement la sélection naturelle. Elle réalise en fait l'accommodation de l'organisation assimilatrice que constitue l'espèce, accommodation à défaut de laquelle l'espèce se transformerait, élargirait ses possibles comme dans l'exemple fameux des pinsons des Galapagos.

On parvient ainsi à cette conclusion que, l'espèce ne se maintient invariante qu'au travers de l'équilibre entre assimilation et accommodation. C'est Piaget retrouvé, à partir de Darwin.

Sur ce, observons bien que dans le processus d'accommodation instancié au niveau des rapports de l'espèce à son milieu, il n'est nulle part fait mention d'une quelconque "résistance" du "réel" : l'accommodation est toujours relative aux rapports de codétermination qu'entretiennent entre elles les différentes formes vivantes. Ceci veut dire que le "réel" n'a pas résisté puisque les "variants" vivent, survivent, et se reproduisent ; ils sont viables. Reste qu'il y a quand même accommodation, au niveau de l'espèce, car, relativement aux autres, ses membres se sont moins reproduits. Tout est là. Nulle réalité, nulle nécessité n'est venue s'imposer "en soi". Ce sont les relations, de compétition, de coopération entre les individus, c'est-à-dire les éléments de l'organisation spécifique, comme les relations - de compétition, de prédation, etc. - entre les espèces, c'est-à-dire les éléments de l'organisation écosystémique qui conditionnent l'avenir de chaque organisation en particulier.

On peut remarquer de plus, qu'à l'instar de l'évolution biologique - qui, dans la perspective écosystémique esquissée plus haut, apparaît comme un processus de venue à la cohérence entre les différentes espèces, constitutives d'une biocénose l'évolution de l'organisation cognitive, de l'écosystème des schèmes, se présente aussi comme processus de venue à la cohérence. Piaget, formidable observateur, n'avait pas manqué de le relever, dès ses premières études : 
"l'intelligence ne pourrait appréhender aucune donnee extérieure sans certaines fonctions de cohérence (dont le terme ultime est le principe de non-contradiction), de mise en relations, etc., qui sont communes a toute organisation intellectuelle" (1936: 9).

"tandis que les schèmes initiaux ne sont reliés entre eux que grâce à leur substructure réflexe et organique, les schèmes les plus évolués, d'abord primaires, puis secondaires et tertiaires, s'organisent peu à peu en systèmes cohérents grâce à un processus d'assimilation mutuelle sur lequel nous avons maintes fois insiste" (1936:362).

Ce qui précède, c'est-à-dire cette mise en regard du schématisme piagétien et d'une conception écosystémique de l'évolution, a directement répondu aux questions que nous nous étions posées. De fait, ainsi qu'on l'a remarqué, l'obligation d'accommodation n'est plus à rapporter à ce qui serait d'incontournables aspérités du "réel", mais bien plutôt aux relations, aux interactions, que l'organisation entretient avec ses collatérales, ou aussi bien, avec l'organisation englobante. Il faut voir là des processus de co-sélection, ou de co-évolution, homologues à ceux que Darwin tentait de mettre en avant pour expliquer l'origine des espèces. Autrement dit, un schème peut parfaitement "délirer" et s'assimiler "tout l'univers" tant qu'il reste peu ou pas couplé à d'autre schèmes, tant qu'il a du "jeu". Le schème de la succion pourra effectivement s'alimenter fonctionnellement avec tout ce qui pourra se prêter à cette activité tant que le couplage avec le cycle fonctionnel de régulation de la glycémie ne se fera pas sentir. Dès que la faim sera là, le schème de succion verra son champ d'activité considérablement restreint et ne s'exercera plus, finalement, que sur le sein. Nous sommes ainsi amenés à insister sur le facteur cohérence et, ce faisant, à en redécouvrir la présence chez Piaget ou chez Baldwin.

\section{V.1 Pour un relativisme interactionniste conséquent}

On pourrait juger inconvenante cette prétention à "redécouvrir" une conception déjà avancée par un auteur, ce qui revient à prétendre lire cet auteur plus sûrement qu'il ne se lisait lui-même. Cette conclusion - qui serait tout à fait banale dans un contexte herméneutique - me paraît inévitable dans la mesure où Piaget n'a jamais su s'arrêter sur ce seul facteur de cohérence, de 
coévolution ou codétermination entre schèmes, s'étant toujours empêtré dans l'appel aux perturbations suscitées par les singularités de l'objet. Et cela, jusque dans ses dernières versions de la théorie de l'équilibration des structures cognitives, qui, soulignons-le au passage, n'avait pas d'autre objectif que de rendre compte de cette cohérence toujours plus grande que l'on peut observer au cours du développement de l'organisation cognitive. Pour son malheur, me semble-t-il, Piaget voulait aussi défendre l'idée de l'autonomie de ce processus de développement, ce qui, par la force des choses, oblige à se distancier du strict relativisme interactionniste soutenu par ailleurs. Piaget avait certainement pressenti la difficulté, mais, soucieux de toujours offrir une image cohérente et, pensant sans doute résoudre la question plus tard, il s'autorisait souvent certaines facilités, proposant des métaphores à la place des définitions, des exemples à la place des explications et rendait ainsi son discours extrêmement difficile à entamer.

Pour s'en convaincre, il n'est que de revenir sur une de ses présentations de l'obligation d'accommodation du schème maintenant que nous sommes pleinement en mesure d'apprécier les effets quelque peu hypnotiques de l'autorité du Maître ${ }^{3}$ :

"(la) tendance à l'application répétée et à la généralisation du schème présente des limites..Ces limites sont imposées par l'expérience (résistance des objets à leur assimilation) et il y a là une nouvelle source d'apprentissage : tout ce qui est vu ne peut pas être saisi ni sucé, ni entendu, etc. On peut citer l'exemple célèbre de la lune, si souvent invoqué ou au contraire contesté, mais que nous avons contrôlé sur un de nos enfants : l'enfant tend les bras vers la lune, après quoi l'activation du schème de la préhension faiblit et disparaît." (Piaget \& Gréco, 1959 : 43).

Où, quand, comment l'objet lune a-t-il résisté aux tentatives d'assimilation de la part du sujet ? Cet exemple est non seulement savoureux mais, très instructif, en ce que l'on voit bien d'une part le "réel" de référence, celui de l'adulte qui a son objet "lune" et d'autre part l'activité déployée par l'enfant. Le sujet s'accommode, c'est un fait. Prétendument, ce serait à cause des résistances de l'objet, mais, de fait, il n'a jamais rencontré le réel "en soi", ni même l'objet "lune" conçu par les adultes, il a tout

${ }^{3}$ C'est bien ainsi que Piaget était présenté par ses collaborateurs (cf. Inhelder, 1977). On s'adressait aussi à lui en donnant du "Patron" ! 
simplement les bras un peu courts pour cela! Que s'est-il donc passé ? Le schème de la vision n'a tout simplement pas pu s'assimiler complètement le schème de la préhension, leur relation d'assimilation réciproque s'est vue accommodée. Finalement, c'est toute l'organisation qui peut être jugée plus cohérente au sens où une "vérité" toute relative à l'organisation - celle qui veut que l'on ne puisse saisir tout ce que l'on voit - s'est vue ébauchée dans l'organisation, par l'accommodation de la relation vision/préhension. Il sera toujours loisible à qui le désire de dire que l'organisation cognitive de l'enfant a commencé à s'adapter à la réalité, mais ce serait négliger le fait qu'il s'agit de "sa" réalité d'entité à bras courts - et que ce soit aussi la nôtre ne la rend pas objective, ou éternelle pour autant ; rappelons qu'en 1969 on a su montrer que la lune pouvait tout à la fois être vue et saisie. Il faut bien repérer ici le relativisme interactionniste qu'introduit cette observation. L'enfant n'apprend que ce que son organisation lui permet de concevoir.

Devrait-on en conclure qu'il nous faut "absenter" le réel ? Non, certes pas, car ce serait reconduire implicitement l'idée fallacieuse et aporétique d'une coupure entre l'organisation et son milieu. Il convient au contraire de s'en tenir à l'idée de la connexité organisation/milieu mise en avant dans la définition du cycle assimilateur. Toute la difficulté réside dans la nécessité de réaffirmer cette position à tous les niveaux que l'on voudra considérer dans l'organisation hiérarchique du vivant, alors qu'il est si facile de se laisser aller à nos anciens schémas, consciemment ou inconsciemment, pour réintroduire la coupure ici ou là.

\section{Eléments de conclusion}

Faire référence, comme Piaget, à des pressions du milicu sous forme de résistances des objets à l'assimilation est, non seulement, contradictoire avec un relativisme interactionniste conséquent mais c'est aussi tout à fait superflu puisque cette dernière conception suffit amplement à expliquer l'adaptation d'un schème. Cette adaptation du schème peut effectivement être vue sur le mode d'une co-adaptation découlant de la codétermination ou cosélection instaurée entre les schèmes par leurs interactions. Il n'y a 
là, en définitive, qu'un aspect inévitable du fonctionnement concomitant des divers schèmes d'une organisation. Chacun de ces schèmes tendant à s'élargir et à s'assimiler les autres schèmes - le schème de la préhension, par exemple, en venant à "s'alimenter" au travers du schème de la vision -, ces tendances assimilatrices hégémoniques, nécessairement opposées, "s'équilibrent" réciproquement et instaurent donc, toutes pour chacunes, une accommodation réciproque, c'est-à-dire une cosélection. C'est, toute choses égales par ailleurs, ce que l'on peut observer en biologie de l'évolution, au niveau de ce que l'on pourrait appeler l'équilibration des "structures" biocénotiques 4 .

\section{VI.1 De l'ontologie}

On pourrait s'empresser, avec raison, de reconnaître dans ce qui précède une problématique des relations tout/parties amplement mise en avant par Piaget, et par de nombreux courants de pensée avant lui mais, on ne saurait l'y réduire. Effectivement, on peut parfaitement s'intéresser aux relations tout/parties dans un contexte purement synchronique comme symbolique alors que la perspective de Piaget était d'abord génétique, c'est-à-dire, et diachronique, et sub-symbolique. Il s'agissait pour lui de penser la genèse du symbole et pas seulement d'en étudier le traitement. C'est pourquoi j'insiste sur le fait que le "Piaget cohérent" que nous avons découvert en l'enracinant dans Darwin ne peut être vraiment compris que dans le cadre d'un ontologie processuelle, où rien ne dispose de la permanence idéelle d'une catégorie, d'un type logique. Le cadre systémique incontournable dans lequel nous devons opérer rend, à terme, illusoire toute volonté de tenir quoi que ce soit pour "objectif" c'est-à-dire, disposant "en soi" de la permanence généralement attribuée aux objets, dans le cadre de l'ontologie "classique". Comme le rappelle très bien Paul Weiss :

${ }^{4}$ Notons au passage que ces "structures" présentent une évolution rythmée, manifestant des stades de relative stabilité, entrecoupés de phases critiques - les radiations adaptatives - au cours desquelles s'effectue l'essentiel du processus évolutif. Ces dernières pourraient fort bien être vues comme processus d'équilibration (majorante ?) ponctuant des stades relativement "équilibrés". D'ailleurs, la théorie des paléontologues Eldredge et Gould qui insiste sur ces phénomènes s'appelle précisément "la théorie des équilibres ponctués". 


\begin{abstract}
"un flux permanent à toutes les échelles exclut les modèles de stockage classiques du type des engrammes et impose les modeles de type reconstructions continuelles" (Inhelder, $1977: 44$ )
\end{abstract}

C'est précisément l'objet du débat le plus actuel en biologie que de savoir si l'espèce ne devrait pas, elle-même, être considérée comme un individu plutôt que comme une simple classe (logique) d'individus du niveau hiérarchique inférieur, les organismes. Cette perspective, qui ne cesse de gagner du terrain, se donne donc un objet à l'ontologie fragile, puisqu'il est sans clôture topologique, et que sa stabilité dépend de ses modalités d'interaction, dans la mesure où ces dernières permettront ou non sa perpétuation. Ces questions ne sont pas nouvelles - les perspectives "organicistes" ne datent pas d'hier -, mais, il est patent qu'elles attendent toujours une conceptualisation sérieuse. Les New Ways of Ontology de Hartmann sont, me semble-t-il, restés sans lendemains, tout comme les holons de Koestler ou les intégrons de Jacob, maintes fois cités mais, à ma connaissance, jamais retravaillés fructueusement. Sans pouvoir, ici, contribuer véritablement à concevoir une ontologie processuelle conséquente, cohérente, il me semble tout de même important de souligner combien l'ontologie piagétienne fondamentale, que l'on peut et doit caractériser par le seul concept de schème, semble convenir à une telle perspective. Outre sa parcimonie, outre le formidable éventail de situations dans lequel Piaget nous la fait découvrir, elle a - avec les notions d'assimilation, accommodation, équilibration - l'avantage d'être d'emblée équipée des outils conceptuels nécessaires à la modélisation d'un univers où rien n'est statique, hormis ce que nous décidons arbitrairement de voir tel, un univers où rien n'est autonome, hormis ce que nous décidons arbitrairement de voir tel.

Singulièrement, le principe moteur de toute la trajectoire piagétienne se trouve être son inébranlable conviction quant à l'autonomie du processus de construction de nos connaissances. Ces dernières, pour n'être pas a priori puisqu'elles procèdent d'une genèse, n'en relèveraient pas moins des nécessités a priori qui déterminent les processus de construction de ces connaissances. Cette préconception repérée, on comprend que, malgré le bel outillage conceptuel dont il disposait, il soit allé s'enferrer dans l'appel aux perturbations. On comprend aussi qu'il 
se soit quelque peu offusqué de voir Lorenz se qualifier de néokantien tout en faisant appel à la sélection naturelle - et donc, aux mutations aléatoires - pour expliquer comment le vivant accumule des connaissances sur le milieu. Le caractère nécessaire des connaissances élaborées ayant de la sorte totalement disparu, Piaget voyait là, plutôt, une trahison de Kant. De là vient aussi son souci constant d'éviter les aspects sélectifs dans l'explication des processus de construction des connaissances car ils seraient porteurs, à ses yeux, d'une inadmissible tare : celle de faire découler nos connaissances, même logico-mathématiques, d'un processus où l'environnement jouerait un rôle déterminant. Alors qu'il conviendrait, au contraire, d'expliquer leur autonomie, c'està-dire, le fait qu'elles se construisent "en logique", nécessairement.

Ce préjugé anti-sélectionniste a tenu Piaget à l'écart d'une perspective populationnelle, c'est-à-dire, à l'écart d'une ontologie radicalement processuelle alors que sa conception "schématique" en constitue, me semble-t-il, le meilleur fondement. Directement ou indirectement, cela a engendré nombre de paradoxes qui émaillent le discours piagétien, tout spécialement au sujet de "l'accord de la pensée avec les choses" dont Piaget n'a finalement plus rien à nous dire, si ce n'est pour suggérer que :

"le sujet est lui-même un objet physico-chimique [...] son harmonie avec le réel vient de ce qu'il procède du réel et qu'il est né de la physicochimie. (Inhelder, $1977: 64$ ),

ce qui, on en conviendra, est un peu court et surprenant de la part d'un théoricien de l'adaptation. Et nous ne dirons rien ici, faute de place, de la singulière argumentation, déployée autour du concept néo-lamarckien de "phénocopie", qui visait à montrer que le génome, lui aussi, se construit, sinon en logique, du moins, nécessairement (Piaget, 1974).

\section{VI.2 La déconstruction du schème}

L'exemple piagétien devrait nous rendre plus vigilants, car, il est clair que nos anciens schémas de pensée "objectivistes" tenteront toujours de s'introduire ici où là. Aussi, pour finir, il me semble opportun de revenir brièvement sur la notion de cycle 
assimilateur, afin de la recadrer dans une perspective "populationnelle", darwinienne, et plus sûrement encore, baldwinienne.

Le cycle assimilateur, tel que Piaget le concevait, pouvait, on l'a vu, faire l'objet d'une lecture "objectiviste", puisque l'on pouvait admettre in fine qu'un cycle adapté puisse se reproduire tel quel sans plus subir d'accommodation. Il me semble qu'une telle éventualité aurait d'emblée paru absurde, si, en lieu et place d'un cycle idéel, Piaget nous avait très explicitement donné à voir une famille de cycles, c'est-à-dire un ensemble potentiel de trajectoires apparentées. Son modèle autorise tout à fait une telle vision, mais, sans doute ne pouvait-clle ĉtre retenue, du fait de l'aspect "sélectionniste" qu'elle induit. Dans un tel cadre, l'accommodation pourrait être aisément traduite par l'idée que certaines trajectoires vont se voir reproduites davantage que d'autres du fait du couplage, des interactions, avec d'autres cycles. On conçoit alors immédiatement que la stabilité d'un schème, c'est-à-dire, la stabilité de l'ensemble de ses trajectoires actuelles résulte de la stabilité, de l'équilibre des interactions entre schèmes couplés. Un schème adapté serait ainsi, nécessairement, un schème en équilibre dynamique. Il y aurait encore beaucoup à dire, à préciser, mais, pour terminer, nous nous contenterons de rappeler qu'il n'y a rien de plus ici que la notion de sélection fonctionnelle invoquée par Baldwin pour dénoter ces processus de sélection intra-organique :

"all new movements which are adaptive or 'fit' are selected from overproduced movements, or movement variations, just as organisms are selected from overproduced variations by the natural selection of those which are fit. This process, thus conceived, is there called 'functional selection', a phrase which emphasizes the fact that it is the organism which secures from all its overproduced movements those which are adaptive and beneficial." (Baldwin, $1902: 87)^{5}$.

5 Il va de soi, mais sans doute vaut-il mieux le rappeler, que "sćlection naturelle" est entendue, ici, sur le mode systémique décrit plus haut. Baldwin a su se tenir proche de Darwin, se gardant de la doctrine néo-darwinienne. 


\section{VI.3 Schème et sub-logique}

A suivre les dernières tendances qui se font jour en I.A. dans le contexte du néo-connexionnisme, on découvre avec plaisir que la remise en question du paradigme computo-symbolique n'est pas simplement méthodologique. Les cadres logiques traditionnels y sont quelque peu malmenés, puisque, par exemple, la classique et rigide conception philosophique type/token - où les token sont les instances, c'est-à-dire les éléments, d'un type logique, d'une classe, et correspondent donc rigoureusement à la définition de leur type - se voit enfin opposer une conception plus souple :

"In fact, the very idea of the type/token distinction need be reconsidered, and perhaps replaced by a couple such as Langacker's scheme/event. Dynamical approaches such as the one we advocate seem to provide this alternative with a coherent modelling methodology. In the traditional framework, tokens are nothing but identical, indexed copies or repetitions of a given type. Here, by contrast, one can think of a scheme as a set of dynamical constraints which partially predetermine the potential trajectories, while an event is primarily a singular trajectory. The difference in geometrical nature between the two reflects a difference in nature between the two cognitives categories which the traditionnal view can only ignore." (Andler, Petitot \& Visetti 1991 : 11).

Les approches dynamiques ont, on le voit bien, une incidence sur l'ontologie qui amène à une convergence frappante avec le schématisme piagétien - convergence déjà esquissée par Asher qui, à l'occasion des 80 ans de Piaget (Inhelder, 1977 : 99), suggérait déjà d'assimiler un schème à un attracteur. Il est simplement regrettable que cela se fasse toujours à reculons, sous la pression des échecs précédents. Il serait, me semble-t-il, avantageux de s'installer d'emblée dans la conception piagétienne qui a tout de même l'avantage remarquable d'embrasser de manière cohérente - c'est-à-dire, sur la base des mêmes invariants - les domaines physico-chimiques, biologiques et surtout psychologiques. Il y aurait quelque inconséquence à négliger cet aspect, car, il faut y insister, l'enjeu essentiel de l'I.A., classique ou néo-connexionniste est avant toute chose ontogénétique et de manière ultime subjectif, puisqu'il s'agit idéalement de recréer une entité aux capacités calquées sur le sujet humain - cf. le test de Turing. 
A l'évidence, les psychologues ont ici un rôle de premier plan à jouer, mais, il faudrait pour cela qu'ils n'abandonnent pas leurs droits d'aînesse sur l'héritage piagétien pour le plat de lentilles du paradigme computo-symbolique, attitude regrettable que leurs complexes vis à vis des sciences "dures" peuvent expliquer mais non justifier.

Luc-Laurent SALVADOR Université René Descartes-Paris V Laboratoire de Psychopathologie

\section{Bibliographie}

Andler, D., Petitot, J. \& Visetti, Y.M. (1991) Dynamical Systems, Connectionism, and Linguistics. Actes du Colloque Interdisciplinaire sur la Compositionnalité dans la cognition et les réseaux neuronaux, Royaumont, mai 1991.

Ashby, W.R. (1962) Principles of the Self-Organizing System, in M. \& G.Yovits, G.T. Jacobi et G.D. Goldstein, Self-Organizing Systems, Spartan Books, Washington.

Atlan, H. (1972) Organisation Biologique et Théorie de l'Information, Hermann, Paris.

Atlan, H. (1979) Entre le cristal et la fumée, Seuil, Paris.

Baldwin, J.M. (1902) Development and evolution, MacMillan, N.Y.

Bastien, C. (1989) Les Modèles de Résolutions de Problèmes, in J.P.Caverni, C.Bastien, P. Mendelhson \& G. Tiberghien (Eds.) Psychologie Cognitive: modèles et méthodes, Presses Universtaires de Grenoble. pp. 27-38.

Bideaud, J. (1989) Logique et Bricolage chez l'enfant, Presses Universitaires de Lille.

Cauzinille, E. (1988) Introduction à la Journée Européenne sur l'Acquisition de Connaissances Structurées, in Cahiers du LAFORIA, $N^{\circ} 69$, p. 65.

Ducret, J.J. (1990) Jean Piaget, biographie et parcours intellectuel, Delachaux et Niestlé, Neuchâtel.

Eigen, M., Gardiner, W., Schuster, P. \& Winkler-Oswatisch, R. (1981) The Origin of Genetic Information, Scientific American, 244, 4, 88-118.

Ganascia, J.M. (1989) L'âme-machine, Seuil, Paris.

Grossberg, S. (1987) Competitive Learning: From Interactive Activation to Adaptive Resonance. Cognitive Science, 11, 26-63.

Gruber, H.E. (1966) Pensée créatrice et vitesse du changement adaptatif : le développement de la pensée de Darwin, in Psychologie et Epistémologie Génétiques, Hommage à Jean Piaget, Dunod, Paris.

Hartmann, N (1953) New Ways of Ontology, Henry Regnery Cie, Chicago.

Inhelder, B., Garcia, R. \& Vonèche, J. (1977) Epistémologie génétique et équilibration, Delachaux \& Niestlé, Neuchâtel.

Jacob, F. (1970) La logique du vivant, Gallimard, Paris. 
Koestler, A. (1979) Janus, Calmann-Lévy, Paris.

Lautrey, J. (1987) Structures et Fonctionnements dans le développement cognitif, Thèse d'etat de Paris V.

Lautrey, J. (1990) Esquisse d'un modèle pluraliste du développement cognitif, in M. Reuchlin, J. Lautrey, C. Marendaz \& T. Ohlmann (eds.) Cognition : l'Individuel et l'Universel, P.U.F., Paris, pp. 185-216.

Le Dantec, F. (1913) La "Mécanique" de la vie, Flammarion, Paris.

Lenay, C. (1989) Le Hasard dans les théories biologiques du XIXe, Thèse doctorale de Paris I.

Monod J. (1970) Le Hasard et la Nécessité, Seuil, Paris.

Patten B.C. \& Auble, G.T. (1981) System theory of the ecological niche, The American Naturalist, Vol 117, pp. 893-922.

Piaget, J. (1936) La Naissance de l'Intelligence chez l'Enfant, Delachaux et Niestle, Neuchâtel.

Piaget, J. (1937) La Construction du Réel chez l'enfant, Delachaux et Niestlé, Neuchâtel.

Piaget, J.(1967) Biologie et Connaissance : essai sur les relations entre les régulations organiques et les processus cognitifs, Gallimard, Paris.

Piaget, J. (1974) Adaptation Vitale et Psychologie de l'Intelligence : sélection organique et phénocopie, Hermann, Paris.

Piaget, J. (1975) L'équilibration des structures cognitives : problème central du développement, PUF, Paris.

Piaget, J. \& Greco, P. (1959) Apprentissage et Connaissance, PUF, Paris.

Pitrat, J. (1990) Métaconnaissances, Hermès, Paris.

Prigogine, I. \& Glansdorff, P. (1971) Structure, stabilité et fluctuations, Masson, Paris.

Salvador, L-L. (1992) Evolution et Herméneutique, vers une approche écosystémique de la cognition, Revue Internationale de Systémique, à paraître.

Simon, H. \& Kaplan, C.A. (1989) Foundations of cognitive science, in M. Posner(ed.) Foundations of Cognitive Science, MIT Press, Cambridge, Mass.

Tiberghien, G. (1986) Psychologie Cognitive, Science de la Cognition et Technologie de la Connaissance, in J.L. Le Moigne (éd.) Intelligence des Mécanismes, Mécanismes de l'intelligence, Fayard, Paris.

Tiberghien, G. (1992) Les Sciences Cognitives : un nouveau programme de recherche ? in L. Sfez (éd.) Dictionnaire Critique de la Communication, PUF, Paris.

Uexküll, J.Von (1926) Theoretical Biology, Kegan, Paul, Trench, Tubner, London.

Uexküll, J.Von (1965) Mondes Animaux et Monde Humain, Denoël, Paris.

Varela, F. (1989) Autonomie et connaissance, Seuil, Paris 